\title{
Minimizing of current harmonics for electrical power system station by controlled current source
}

\author{
Dr. Jaber Ghaib Talib \\ Al-mostakbal University College/ Babylon, Iraq
}

\begin{abstract}
:
In this optional method, controlled current source CCS with controlled voltage source CVS is used to increase the high power system station acts. The traditional system causes high odd and even harmonics for current and voltage that it leads to decreasing the system efficiency. The output sinewave voltage and current is distorted with more unstable. Therefore, the best solution for this critical situation, CCS with CVS controller are used to develop the efficiency by reducing the steady state error, decreasing the overshoot with undershoots for system. The results are completed depending on Matlab Simulink with toolbox. The system with two controllers has the consultant over conventional system with increase the active power and minimizing losses power by limitation the harmonics.
\end{abstract}

Key words: power system station, CVS and CCS controller, current and voltage harmonics, Matlab.

\section{Introduction:}

High power system stations are the main power for distribution and transmission power from the electrical generation system. These distributed stations have many problems to solve it to enhance the active power [1]. Therefore, the quality of power is suggested by some author to increase the efficiency [2] [3]. In addition, the harmonics is growing because of divergence between the frequency of signal with voltage supplied to system. The active power is projected by expansion control approach to optimize efficiency [5]. The authors in [6] reported simple method that focusing on active power. Multiple adjustable current is recycled to enhance the harmonics of current [7]. Also, the shunt active filter is established to enhance the system of power station [8]. A hybrid technique based power filter and thermistors are also presented [9]. The passive and active filters are applied to power station to augment the system [10]. Lastly, current control is created to adjustable the voltage and frequency deviation by using advancement method [11] 12].

In this method, new contribution is optional to increase the reliability of power station based on controlled voltage and controlled current sources. The main advantage of controller is to track the output current and voltage with input source.

\section{Simulation Results:}

In this method, the inputs to controller are output voltage and output current. The Simulink of CCS with CVS is shown in figure 1 .

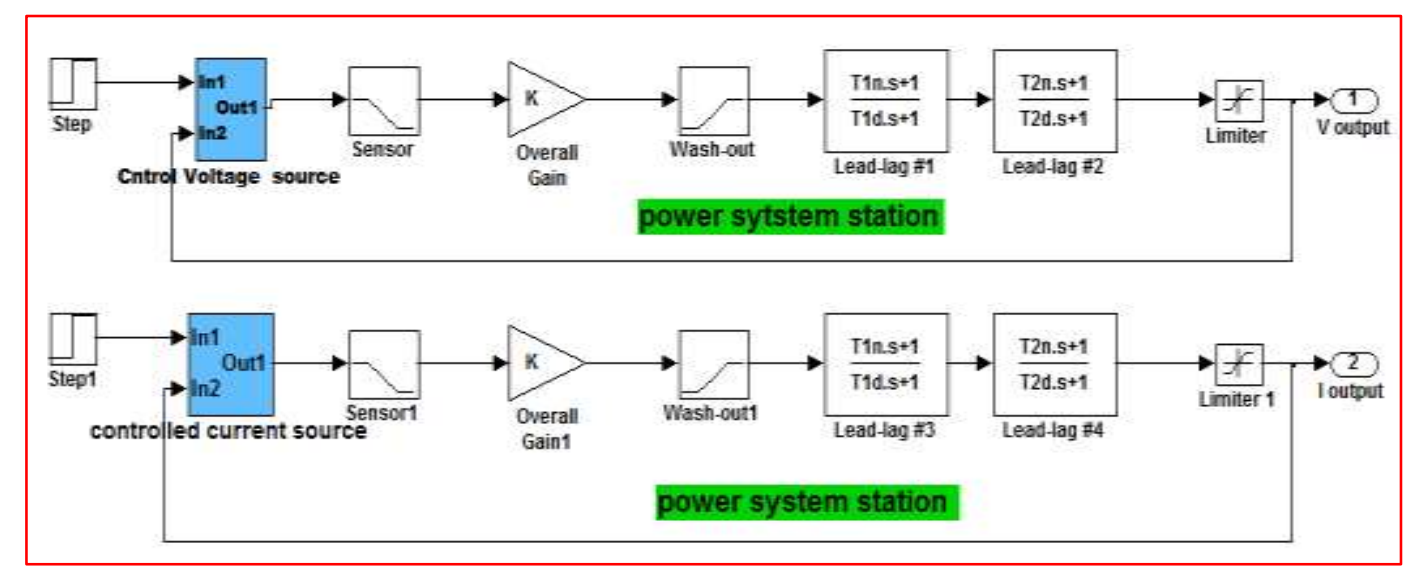

Figure 1: Simulink of CCS and CVS. 
The harmonics of voltage and current in the classical power system station under the loads is very high s shown in figure 2 and figure 3 respectively.

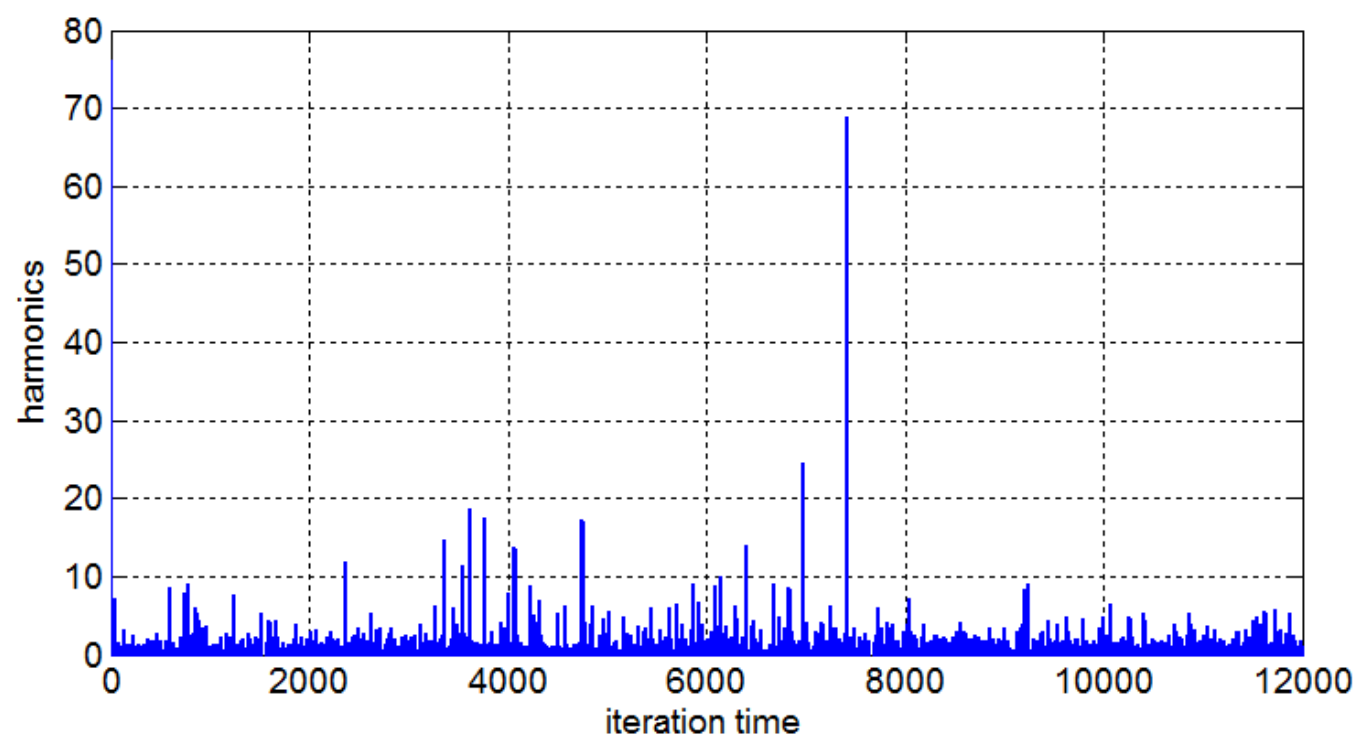

Figure 2: harmonics of current.

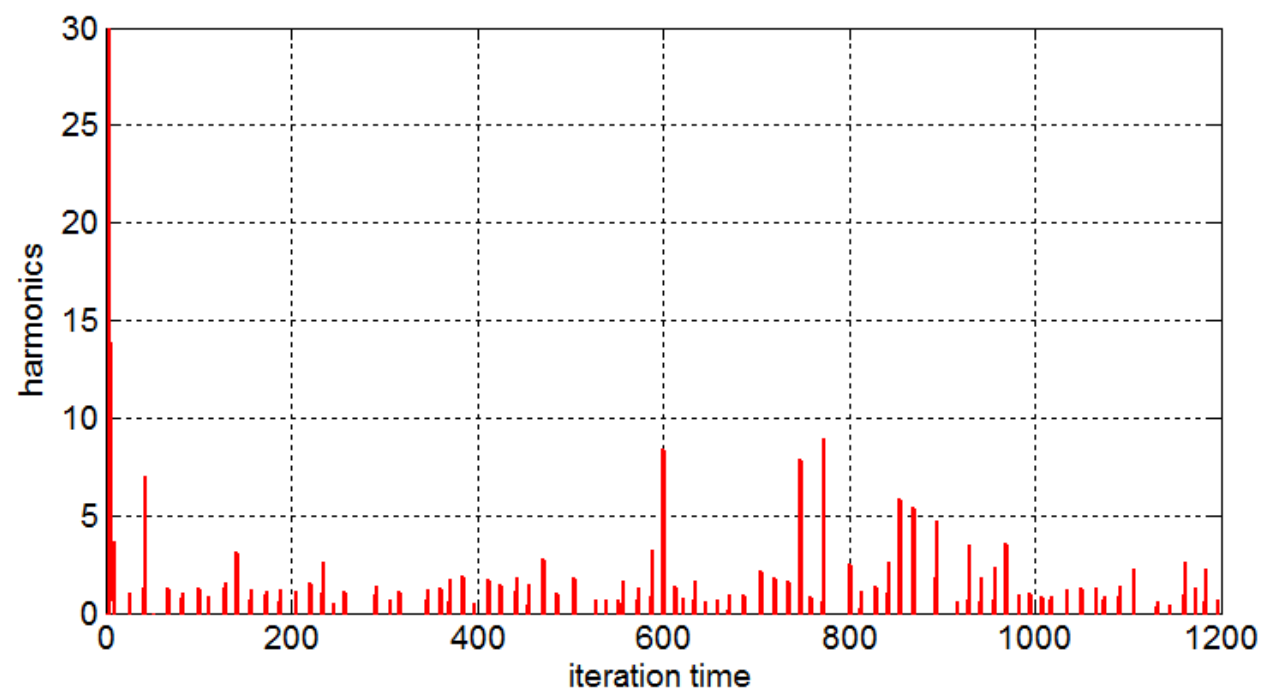

Figure 3: hrmonics of voltage.

In figures 4 and figure 5. When the proposed CCS and CVS are used together in power system station, the harmonics is decreased and reach to zero . 


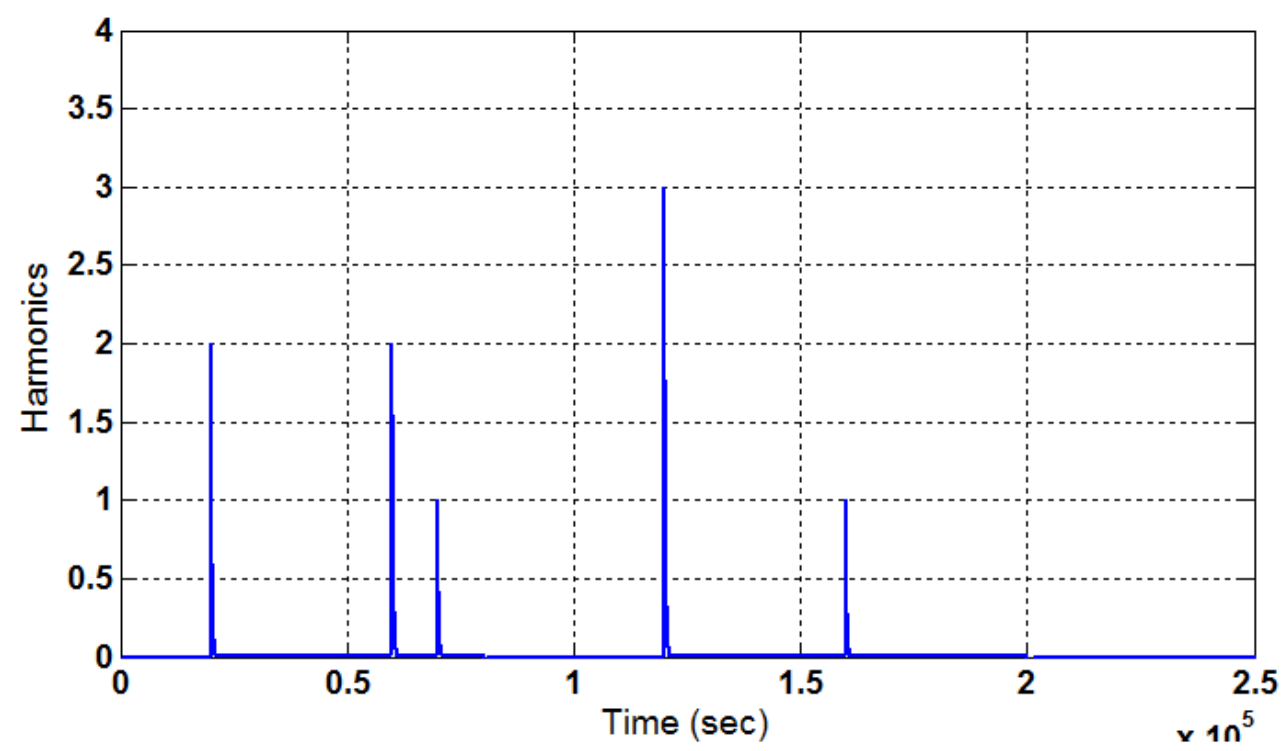

Figure 4: harmonics of current by using CCS

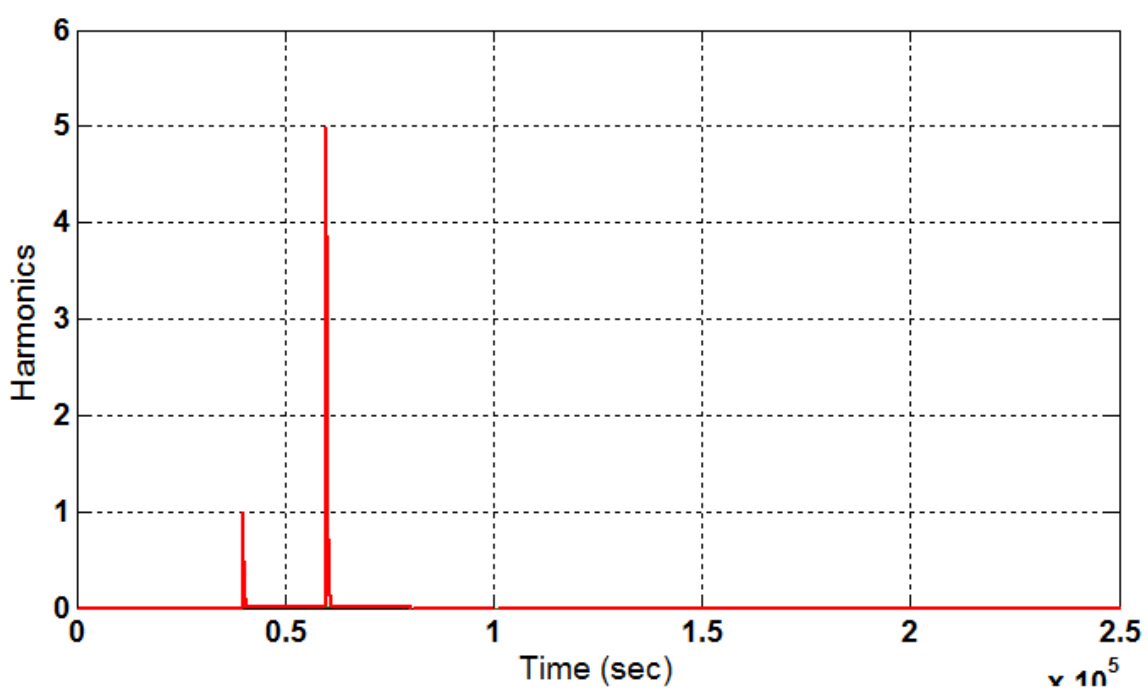

Figure 5: Figure 4: harmonics of voltage by using CVS

Conclusion:

In this method, the CCS and CVS are used to minimize the error between the desired and real output for voltage and current as results to make the system of power station is more reliability and high accuracy for optimize efficiency. Therefore, the losses of copper and iron losses of system will decrease. The target of this method is to make the system fast response and good tracking with input source. Finally, the power station with CCS and CVS is rigged, tough and high ability to control the voltage and current.

\section{References}

1. IEEE Std. 519-1992, "IEEE Recommended Practices And Requirement For Harmonic Control In Electrical Power Systems".

2. Chapman David, "The Cost of Poor Power Quality, Power Quality Application Guide, Copper Development Association, U.K, March, 2001".

3. Santoso, Surya, Dugan, R.C, Mc. Granaghan, M.F., Beaty, H.W, "Electrical Power System Quality", McGraw-Hill, Second Edition, 2003".

4. Mauricio Angulo, "IEEE Transactions on Industrial Electronics, Vol.60. 7 July 2013".

5. Czarnecki, I.S., "What is Wrong with Budeanu's Concept of Reactive and Distortion Power and Why it should be Abandoned" IEEE Transactions on Instrumentation and Measurement, Vol. 36, 3 September,1987.

6. Francisco C. De La Rosa, "Harmonics and Power System” Sun Francisco, June, 2005. 
7. Emanuel, A.E..," On the assessment of Harmonic Pollution"” IEEE Transactions on Power Delivery, Vol. 10, no. 3, July 1995, pp. 767-772.

8. Mostafa S. Hamad, Mahmoud I. Masoud, et al., "A shunt active power filter for a medium voltage 12-pulse current source converter using open loop current compensation." In IEEE Transactions on Industrial Electronics, Vol.61, No.11, November 2014 on P (5840-5850).

9. Salem Rahmani, Abdelhamid Hamadi, et al., "A combination of shunt hybrid power filter and thyristor controlled reactor for power quality." IN IEEE Transactions on Industrial Electronics, Vol.61, No. 5, May 2014 On P (2152-2164).

10. Jinwei He, Xiongfei Wang, et al., "Active harmonic filtering using current-controlled, grid connected DG units with close- loop power control." In IEEE Transactions on Power Electronics, Vol.29, No.2, February 2014 on P (642-653).

11. Parag Kanjiya, Vinod Khadkikar, et al., "Optimal control of shunt active power filters to meet IEEEstd.-519 current harmonics constraints under non-ideal supply condition." In IEEE Transactions on Industrial Electronics 2014.

12. Quoc-Nam Trinh, et al., "An advanced current control strategy for shunt APF." In IEEE Transactions on Industrial Electronics, Vol.60, No.12, Dec 2013 